Artículo Especial

\title{
Síndrome de ovario poliquístico y complicaciones metabólicas: más allá del exceso de andrógenos
}

\author{
Andrea Facio-Lince García. 1a, María Isabel Pérez-Palacio. 1a, Juliana Lucia Molina- \\ Valencia. ${ }^{1 a}$, Lina María Martínez-Sánchez. ${ }^{1 b}$ \\ ${ }^{1}$ Grupo de Investigación en Ginecología y Obstetricia, Facultad de Medicina, Universidad Pontificia Bolivariana, Sede \\ Central Medellín, Circular 1 No. 70-01, Medellín, Colombia.
}

\footnotetext{
a Alumnas, Facultad de Medicina, Universidad Pontificia Bolivariana, Sede Central Medellín, Circular 1 No. 70-01, MedeIlín, Colombia. ${ }^{\mathrm{b}} \mathrm{MSc}$, Docente Titular, Facultad de Medicina, Universidad Pontificia Bolivariana, Sede Central Medellín, Circular 1 No. 70-01, Medellín, Colombia.
}

\section{RESUMEN}

El síndrome del ovario poliquístico (SOP) es la alteración endocrinológica más común que afecta a las mujeres en edad reproductiva con estudios que reportan una prevalencia de hasta un $21 \%$. Existen diferentes criterios de diagnóstico que describen diferentes fenotipos en las pacientes. EI SOP trae grandes implicaciones en la salud femenina ya que se ha relacionado con alteraciones endocrinológicas, metabólicas y de fertilidad. Actualmente hay mayor conocimiento sobre la patogénesis de la enfermedad y se han relacionado nuevos marcadores bioquímicos tanto en mecanismos fisiopatológicos propios del SOP, como de enfermedades asociadas, permitiendo mejor identificación y manejo de esta población, lo que implica un avance para el desarrollo de posibles terapias y métodos diagnósticos más eficaces.

\section{PALABRAS CLAVE: Síndrome del ovario poliquístico, síndrome metabólico, resistencia a la Insulina, hiperandrogenismo}

\section{SUMMARY}

Polycystic ovarian syndrome (PCOS) is the most common endocrine disorder, it affects mostly women of reproductive age and some studies have reported that up to $21 \%$ of women present this syndrome. There are different diagnostic criteria for PCOS that characterize these patients into one of various phenotypes. This disorder has great implications on women's health because it has been linked to other metabolic, endocrine and fertility disorders. Due to recent investigations, there has been a surge of new information about the pathogenesis of this disease, which in turn has helped explore new biochemical markers and pathophysiological mechanisms of PCOS and associated diseases, thus allowing a clearer identification and better management of these patients. This new information can lead to a breakthrough in the development of potential therapies and more effective diagnostic methods.

KEY WORDS: Polycystic ovary syndrome, metabolic syndrome, insulin resistance, hyperandrogenism 


\section{INTRODUCCIÓN}

En el año 1935 Stein y Leventhal describieron por primera vez pacientes con Síndrome de Ovario Poliquístico (SOP), en mujeres con amenorrea, hirsutismo, obesidad e histología de ovario poliquístico $(1,2,3)$. En la actualidad representa la alteración endocrina más frecuente en mujeres en edad reproductiva. Múltiples grupos de trabajo han establecido diferentes criterios diagnósticos ya que se trata de un síndrome muy heterogéneo y las manifestaciones clínicas son muy diversas entre la población. Se cuenta con tres grupos principales de criterios diagnósticos, esto explica el amplio rango en la prevalencia reportada en la literatura, desde $6 \%$ hasta $21 \%(4,5)$.

La variabilidad en la presentación clínica está influenciada por factores genéticos, ambientales y endocrinológicos (1). Se caracteriza por la presencia de ovario poliquístico, amenorrea, hiperandrogenismo y alteraciones metabólicas principalmente $(5,6)$.

Esta patología se ha relacionado con alteraciones metabólicas, cardiacas, sexuales, psicológicas y mayor riesgo de cáncer de mama y endometrio (7-10).

Debido a la influencia en la morbi-mortalidad femenina de las complicaciones metabólicas y cardiovasculares se desarrolla este artículo con el fin de resaltar la importancia de un adecuado enfoque y manejo para modificar e intervenir los factores de riesgo en estas mujeres.

\section{Hiperandrogenismo}

La alteración hormonal más destacada por sus repercusiones es el hiperandrogenismo, el cual hace a las pacientes más susceptibles para desarrollar complicaciones a corto y largo plazo. En pacientes con SOP se ha asociado a un peor perfil metabólico y de sensibilidad a la insulina en comparación con las pacientes sanas y las pacientes sin hiperandrogenismo que también padecen SOP, sin embargo estas últimas pacientes presentan niveles de insulina mayores que las mujeres sanas, por lo que no se descarta el riesgo metabólico en este grupo de pacientes $(11,12)$. Incluso se ha visto que a pesar de tener niveles de andrógenos dentro de los límites normales presentan valores en el perfil lipídico mayores que las mujeres sanas $(12,13)$.

Las guías de práctica clínica actuales de la Sociedad de Endocrinología hablan del hiperandrogenismo bioquímico como un aumento en el nivel de andrógenos, el cual, típicamente incluye valores elevados en la testosterona total, biodisponible o libre, pero no hace referencia a los demás andrógenos y su importancia en el diagnóstico o las implicaciones. Tampoco se habla del método adecuado y por consiguiente no existe un valor de referencia (14).

La testosterona ha sido el andrógeno más estudiado, se ha demostrado que guarda una relación directa con la gravedad del síndrome metabólico independientemente de la presencia de SOP; las mujeres obesas con hiperandrogenismo tienen más riesgo de desarrollar síndrome metabólico, dislipidemia y alteraciones en la glicemia (15). Por el contrario, la androstenediona, al parecer tiene un efecto protector en el perfil lipídico y guarda una relación inversa con la resistencia a la insulina. Las pacientes con SOP tienen niveles de andrógenos más altos que las mujeres que no lo tienen, sin importar la presencia de síndrome metabólico (16).

\section{Resistencia a la insulina}

Es definida como la incapacidad de la célula para responder adecuadamente a la señalización de la insulina (17). Esto se hace evidente, por el estado de hiperglucemia, producto de la disminución de la absorción de glucosa en el músculo estriado, sumado a la producción endógena de ésta en el hígado. En un espectro más amplio, la disrupción en la señalización de la insulina lleva a una alteración en el metabolismo de lípidos y proteínas, aumentando la lipólisis, disminuyendo la síntesis proteica y la masa pancreática entre otras múltiples alteraciones, las cuales constituyen factores de riesgo para el desarrollo del síndrome metabólico y la enfermedad cardiovascular (18).

El tejido adiposo es pieza clave en la patogenia de la resistencia a la insulina y se encuentra altamente relacionado con el sobrepeso y la obesidad; los adipocitos hipertróficos disfuncionales, ubicados principalmente en el tejido adiposo visceral y subcutáneo, poseen una alta actividad lipolítica resultando en un aumento de los ácidos grasos libres que contribuyen a la resistencia hepática a la insulina, así como una secreción alterada de adipoquinas y citoquinas, las cuales participan en los procesos de señalización de dicha hormona (18).

Se han observado niveles elevados de factor de necrosis tumoral $\alpha$ (TNF $\alpha$ ) en pacientes obesas y también en pacientes con SOP independiente del índice de masa corporal (IMC). En pacientes obesos y diabéticos se ha observado la relación del TNFa con la resistencia a la insulina, producto de la fosforilación de serina en el receptor de sustrato de insulina tipo 1 (IRS-1). Este hecho conlleva a una disminución del receptor transportador de glucosa GLUT $4(17,19)$.

La resistencia a la insulina conduce a la hiperinsulinemia, debido a una secreción compensatoria y a una menor depuración de dicha hormona, producto de una captación reducida por parte de los tejidos (18). El aumento en las concentraciones 
de insulina lleva a una mayor producción de andrógenos debido a que aumenta la frecuencia de los pulsos de la hormona luteinizante (LH) producidas por una estimulación de la hormona liberadora de gonadotropinas $(\mathrm{GnRH})$ en el hipotálamo. La insulina también aumenta la androgenemia por efectos directos en el ovario, aumentando la transcripción de la proteína esteroidogénica aguda, implicada en el transporte de colesterol dentro de la mitocondria. La hiperandrogenemia es el causante de las manifestaciones típicas del SOP y a su vez empeora la resistencia a la insulina, aumentando los ácidos grasos libres circulantes. Además, los andrógenos parece que tienen un efecto en la disminución de la sensibilidad de la insulina en las fibras muscular tipo I (17).

\section{Diabetes mellitus tipo 2}

EI SOP es considerado por la Federación Internacional de Diabetes y la Asociación Americana de Diabetes como un factor de riesgo no modificable para el desarrollo de diabetes mellitus tipo 2, con un riesgo de 5 a 10 veces mayor que el normal y una prevalencia aproximada del $7 \%(20,21)$. La resistencia a la insulina está presente hasta en el $80 \%$ de las mujeres afectadas y la disfunción de las células $\beta$ del páncreas se relacionan fuertemente con la aparición de diabetes mellitus tipo 2; ambos factores se encuentran presentes en la fisiopatología de este síndrome $(22,23,24)$.

Un estudio que siguió a 1.127 mujeres norteamericanas de raza blanca y negra, procedentes del estudio que evaluó el riesgo cardiovascular en adultos jóvenes (CARDIA, CoronaryArteryRisk In Young Adults) durante 18 años, sugiere que las pacientes con SOP, diagnosticadas con los criterios del Instituto Nacional de Salud $(\mathrm{NIH}$, National Institutes of Health), presentan más riesgo de desarrollar diabetes mellitus tipo 2 y dislipidemia que las pacientes sanas; este riesgo puede ser hasta 3 veces más en pacientes afectadas sin sobrepeso comparado con mujeres sin SOP y sin sobrepeso (25).

Las condiciones que hacen más propensas a estas mujeres para desarrollar alteraciones en el metabolismo de los carbohidratos son la historia familiar de diabetes, la edad, la obesidad y especialmente el exceso de tejido adiposo visceral. Un estudio que evaluó la relación de la historia familiar de diabetes mellitus tipo 2 con la presencia de esta en pacientes con SOP, arrojó como resultado que el $36 \%$ de las pacientes tienen historia familiar positiva; esta relación aumenta a más del $40 \%$ cuando las pacientes presentan prediabetes o diabetes. (26).

El parámetro de laboratorio para el estudio del metabolismo de carbohidratos que se ve alterado con mayor frecuencia es la glicemia a las dos horas luego de una prueba de tolerancia a la glucosa. La glicemia en ayunas puede estar en los valores normales aun cuando existe una alteración de las demás mediciones. Por esto se recomienda la prueba de tolerancia a la glucosa para hacer el tamizaje de las adolescentes y adultas con SOP $(14,28,29)$.

\section{Dislipidemia}

Se estima que cerca del $70 \%$ de las mujeres con SOP presentan por lo menos una alteración en el perfil lipídico, siendo más prevalente en las mujeres obesas. Se observa en esta población niveles más altos de lipoproteínas de baja densidad (low density lipoprotein, LDL) y triglicéridos (TG), y una disminución de las lipoproteínas de alta densidad (high density lipoprotein, HDL) sin importar el IMC.

La resistencia a la insulina y la hiperinsulinemia compensatoria muestran una relación positiva con las cifras de colesterol total, LDL y TG, y una relación negativa en las cifras de HDL, esto debido a una estimulación de la lipolisis y una expresión alterada de la lipoproteína lipasa y la lipasa hepática. El hiperandrogenismo parece tener una relación independiente con la dislipidemia $(27,30)$.

\section{Síndrome metabólico}

La prevalencia del síndrome metabólico (SM) ha aumentado en la población general, con reportes de hasta un $25 \%$ en Europa y Norteamérica, incrementando la posibilidad de desarrollar diabetes tipo 2 y enfermedades cardiovasculares, al catalogarse como un poderoso factor de riesgo para dichas patologías. Su asociación con el SOP ha presentado una frecuencia variable, con una menor prevalencia en las mujeres del Este Asiático y una mayor, en las mujeres africanas, hispanas y del Sur de Asia $(31,32)$.

Las pacientes con SOP que dentro de su fenotipo cuentan con hiperadrogenismo tienen peores estados metabólicos, con niveles altos de testosterona libre y asociada a androstenediona elevada, presentan mayor prevalencia de síndrome metabólico, con 18 y $29 \%$ respectivamente, comparadas con las mujeres control que mostraron un $7,7 \%$ $(13,14)$.

La obesidad puede influenciar la severidad del síndrome metabólico, cerca del $50 \%$ de las mujeres con SOP son obesas. El incremento de la grasa corporal se relaciona con el desarrollo de resistencia a la insulina y viceversa, ambas implicadas en el desarrollo del síndrome metabólico $(27,33)$. Por otra parte la hiperinsulinemia promueve la secreción de andrógenos aumentando el riesgo de enfermedad hepática y aterosclerótica, además de perpetuar la resistencia a la insulina $(34,35)$.

Esta agrupación de anomalías metabólicas que se producen simultáneamente en un individuo 
parece conferir un riesgo cardiovascular adicional sustancial por encima de la suma del riesgo asociado con cada anormalidad (36).

\section{Enfermedad cardiovascular y cerebrovascular}

EI SOP se ha relacionado con la aparición de enfermedad cardio-cerebro vascular en mujeres de edad fértil. Como se ha mencionado anteriormente las mujeres con SOP tienden a tener hiperinsulinemia y dislipidemia, dos factores importantes en el desarrollo de alteraciones vasculares (37).

Se han dilucidado 2 marcadores importantes para el desarrollo de enfermedad cardiovascular en mujeres con SOP. Uno es la calcificación de las arterias coronarias (CAC) y el otro es el grosor íntima-media carotidea (CIMT), estos marcadoresse han encontrado elevados en mujeres con esta patología (37).

Las mujeres con SOP no solo tienen alterados los marcadores de enfermedad cardiovascular sino que también, debido al aumento en la prevalecía de síndrome metabólico y enfermedad coronaria en estos individuos, existe 2 veces más riesgo de desarrollar un evento cardiaco. Estas pacientes demuestran niveles mayores de CIMT comparadas con mujeres sin SOP, esto indica que las mujeres con SOP desarrollan aterosclerosis prematura, que a su vez contribuye como factor de riesgo cardiovascular $(38,39)$.

Varios estudios han demostrado que una de las causas para que las mujeres presenten aterosclerosis prematura es debido a la presencia aumentada de proteína $\mathrm{C}$ reactiva (PCR), un biomarcador inflamatorio que según Hyderali y Mala (37) está involucrado en el proceso aterosclerótico ya que causa una disfunción endotelial importante. También se ha relacionado el SOP con un aumento en el riesgo de tromboembolismo venoso. La formación de trombos se asocia con calcificación venosa, contribuyendo a su vez a la progresión de la aterosclerosis. Un estudio piloto realizado en 90 mujeres con SOP y 35 controles concluyó que la generación de trombina es mayor en mujeres con SOP, y que esto, correlacionado con otros estudios realizados, depende del IMC, colesterol total y grado de inflamación, los cuales se encuentran generalmente aumentados en estas pacientes (39).

\section{CONCLUSIÓN}

EI SOP es una enfermedad endocrina con un compromiso caracterizado por una pérdida de la homeostasis de las hormonas sexuales. Sin embargo, en los últimos años se ha evidenciado el papel que dichas hormonas, principalmente los andrógenos, ejercen sobre la disregulación del metabolismo de otros órganos. Es así, como el hiperadrogenismo promueve un estado hiperinsulinémico, el cual se asocia con diabetes, dislipidemia, riesgo cardiovascular y síndrome metabólico, pero a su vez potencia la producción de andrógenos. Es entonces un círculo vicioso en el que se generan las condiciones necesarias para desarrollar y perpetuar alteraciones endocrinas diferentes a las reproductivas siendo la base para presentar eventos agudos y crónicos de otras enfermedades que van a empobrecer la salud y calidad de vida de las pacientes con SOP. Enfocarse únicamente en las alteraciones reproductivas del SOP es dejar pasar la oportunidad para modificar los factores predisponentes para el desarrollo de otras patologías, un abordaje integral es fundamental para mejorar la salud de las mujeres.

\section{REFERENCIAS}

1. March WA, Moore VM, Willson KJ, Phillips DI, Norman RJ, Davies MJ. The prevalence of polycystic ovary syndrome in a community sample assessed under contrasting diagnostic criteria. Hum Reprod. 2010; 25(2):544-51.

2. Vassilatou E. Nonalcoholic fatty liver disease and polycystic ovary syndrome. World J Gastroenterol. 2014; 20(26):8351-63.

3. Seeber B, Morandell E, Lunger F, Wildt L, Dieplinger $\mathrm{H}$. Afamin serum concentrations are associated with insulin resistance and metabolic syndrome in polycystic ovary syndrome. Reprod Biol Endocrinol. 2014;12:88.

4. Spritzer PM. Polycystic ovary syndrome: reviewing diagnosis and management of metabolic disturbances. Arq Bras Endocrinol Metabol. 2014;58(2):182-7.

5. Hung JH, Hu LY, Tsai SJ, Yang AC, Huang MW, Chen PM, et al. Risk of psychiatric disorders following polycystic ovary syndrome: a nationwide populationbased cohort study. PLoS One. 2014; 9(5):e97041.

6. Cırık DA, Dilbaz B. What do we know about metabolic syndrome in adolescents with PCOS? J Turk Ger Gynecol Assoc. 2014; 15(1):49-55.

7. Barthelmess EK, Naz RK. Polycystic ovary syndrome: current status and future perspective. Front Biosci (Elite Ed). 2014;6:104-19.

8. Banting LK, Gibson-Helm M, Polman R, Teede HJ, Stepto NK. Physical activity and mental health in women with polycystic ovary syndrome. BMC Womens Health. 2014;14(1):51.

9. Aquino $\mathrm{Cl}$, Nori SL. Complementary therapy in polycystic ovary syndrome. Transl Med UniSa. 2014;9:5665.

10. Amato MC, Giordano C. Visceral adiposity index: an indicator of adipose tissue dysfunction. Int $\mathrm{J}$ Endocrinol. 2014; 2014:730827.

11. O'Reilly MW, Taylor AE, Crabtree NJ, Hughes BA, Capper F, Crowley RK, et al. Hyperandrogenemia predicts metabolic phenotype in polycystic ovary syndrome: the utility of serum androstenedione. J Clin Endocrinol Metab. 2014;99(3):1027-36.

12. Jamil AS, Alalaf SK, Al-Tawil NG, Al-Shawaf T. A case-control observational study of insulin resistance and metabolic syndrome among the four phenotypes of polycystic ovary syndrome based on Rotterdam criteria. Reprod Health. 2015;12(1):7. 
13. Lerchbaum E, Schwetz V, Rabe T, Giuliani A, Obermayer-Pietsch B. Hyperandrogenemia in polycystic ovary syndrome: exploration of the role of free testosterone and androstenedione in metabolic phenotype. PLoS One. 2014;9(10):e108263.

14. Legro RS, Arslanian SA, Ehrmann DA, Hoeger KM, Murad MH, Pasquali R, et al. Diagnosis and treatment of polycystic ovary syndrome: an Endocrine Society clinical practice guideline. J Clin Endocrinol Metab. 2013;98(12):4565-92.

15. Valderhaug TG, Hertel JK, Nordstrand N, Dale PO, Hofsø D, Hjelmesæth J. The association between hyperandrogenemia and the metabolic syndrome in morbidly obese women. Diabetol Metab Syndr. 2015;7:46.

16. Tziomalos K, Katsikis I, Papadakis E, Kandaraki EA, Macut D, PanidisD. Comparison of markers of insulin resistance and circulating androgens between women with polycystic ovary syndrome and women with metabolic syndrome. Hum Reprod. 2013;28(3):785-93.

17. Rojas J, Chávez M, Olivar L, Rojas M, Morillo J, Mejías $\mathrm{J}$, et al. Polycystic ovary syndrome, insulin resistance, and obesity: navigating the pathophysiologic labyrinth. Int J Reprod Med. 2014; 2014:e719050.

18. Castro AV, Kolka CM, Kim SP, Bergman RN. Obesity, insulin resistance and comorbidities? Mechanisms of association. Arq Bras Endocrinol Metabol. 2014;58(6):600-9.

19. González F. Inflammation in Polycystic Ovary Syndrome: underpinning of insulin resistance and ovarian dysfunction. Steroids. 2012; 77(4):300-5.

20. Galazis N, Afxentiou T, Xenophontos M, DiamantiKandarakis E, Atiomo W. Proteomic biomarkers of type 2 diabetes mellitus risk in women with polycystic ovary syndrome. Eur J Endocrinol. 2013;168(2):3343.

21. Gambineri A, Patton L, Altieri P, Pagotto U, Pizzi C, Manzoli L, et al. Polycystic ovary syndrome is a risk factor for type 2 diabetes: results from a long-term prospective study. Diabetes. 2012; 61(9):2369-74.

22. Ovalle F, Azziz R. Insulin resistance, polycystic ovary syndrome, and type 2 diabetes mellitus. Fertil Steril. 2002;77(6):1095-105.

23. Allahbadia GN, Merchant R. Polycystic ovary syndrome and impact on health. Middle East Fertil Soc J. 2011;16(1):19-37.

24. Avila MA, Bruno RV, Barbosa FC, Andrade FC, Silva $A C$, Nardi $A E$. Polycystic ovary syndrome: implications of metabolic dysfunction. Rev Col Bras Cir. 2014;41(2):106-10.

25. Wang ET, Calderon-argalit R, Cedars MI, Daviglus ML, Merkin SS, Schreiner PJ, et al. Polycystic ovary syndrome and risk for long-term diabetes and dyslipidemia. Obstet Gynecol. 2011;117(1):6-13.

26. Lerchbaum E, Schwetz V, Giuliani A, ObermayerPietsch B. Influence of a positive family history of both type 2 diabetes and PCOS on metabolic and endocrine parameters in a large cohort of PCOS women. Eur J Endocrinol Eur Fed Endocr Soc. 2014;170(5):72739.
27. Ebrahimi-Mamaghani M, Saghafi-Asl M, Pirouzpanah S, Aliasgharzadeh A, Aliashrafi S, Rezayi N, et al. Association of insulin resistance with lipid profile, metabolic syndrome, and hormonal aberrations in overweight or obese women with polycystic ovary syndrome. J Health Popul Nutr. 2015;33(1):157-67.

28. Hurd WW, Abdel-Rahman MY, Ismail SA, Abdellah MA, Schmotzer CL, Sood A. Comparison of diabetes mellitus and insulin resistance screening methods for women with polycystic ovary syndrome. Fertil Steril. 2011;96(4):1043-7.

29. Weerakiet S, Srisombut C, Bunnag P, Sangtong S, Chuangsoongnoen N, Rojanasakul A. Prevalence of type 2 diabetes mellitus and impaired glucose tolerance in Asian women with polycystic ovary syndrome. Int J Gynaecol Obstet. 2001;75(2):177-84.

30. Azziz R, Carmina E, Dewailly D, Diamanti-Kandarakis $\mathrm{E}$, Escobar-Morreale HF, Futterweit W, et al. The Androgen Excess and PCOS Society criteria for the polycystic ovary syndrome: the complete task force report. Fertil Steril. 2009;91(2):456-88

31. Cauchy F, Fuks D, Zarzavadjian Le Bian A, Belghiti J, Costi R. Metabolic syndrome and non-alcoholic fatty liver disease in liver surgery: The new Scourges? World J Hepatol. 2014; 6(5):306-14.

32. Ladrón de Guevara A, Fux-Otta C, Crisosto N, Szafryk de Mereshian P, Echiburú B, Iraci G, et al. Metabolic profile of the different phenotypes of polycystic ovary syndrome in two Latin American populations. Fertil Steril. 2014;101(6):1732-9.e1-2.

33. Messinis IE, Messini Cl, Anifandis G, Dafopoulos K. Polycystic ovaries and obesity. Best Pract Res Clin ObstetGynaecol. 2015;29(4):479-88.

34. Münzker J, Hofer D, Trummer C, Ulbing $M$, Harger $\mathrm{A}$, Pieber T, et al. Testosterone to dihydrotestosterone ratio as a new biomarker for an adverse metabolic phenotype in the polycystic ovary syndrome. J Clin Endocrinol Metab. 2015;100(2):653-60.

35. Tosi F, Di Sarra D, Kaufman JM, Bonin C, Moretta R, Bonora $\mathrm{E}$, et al. Total body fat and central fat mass independently predict insulin resistance but not hyperandrogenemia in women with polycystic ovary syndrome. J Clin Endocrinol Metab. 2015;100(2):661-9.

36. Alberti KGMM, Zimmet P, Shaw J. Metabolic syndrome a new world-wide definition. A consensus statement from the International Diabetes Federation. Diabet Med. 2006; 23(5):469-80.

37. Hyderali B, Mala K. Oxidative stress and cardiovascular complications in polycystic ovarian syndrome. Eur J Obstet Gynecol Reprod Biol. 2015;191:15-22.

38. De Groot P, Dekkers O, Romijn J, Dieben S, Helmerhorst F. PCOS, coronary heart disease, stroke and the influence of obesity: a systematic review and meta-analysis. Hum Reprod Update. 2011;17(4):495500.

39. Glintborg D, Sidelmann JJ, Altinok ML, Mumm H, Andersen $M$. Increased thrombin generation in women with polycystic ovary syndrome: A pilot study on the effect of metformin and oral contraceptives. Metabolism. 2015; 64(10):1272-8. 